\title{
Del modelo de educación jurídica del siglo XIX en el Perú a un nuevo paradigma de innovación jurídica pedagógica y didáctica para el siglo XXI.
}

Analysis of the Education Model of the 19th Century and Comparing It to the Pedagogical and Didactic Legal Innovation of the 21 st Century

José Pavlov Valdivia-Reynoso, Neil Tejada-Pacheco

Universidad Católica de Santa María, Arequipa, Perú.

\section{INFORMACIÓN}

\section{Historia del Artículo}

Recepción: 31/10/2019

Revisión: 20/11/2019

Aceptación: 02/01/2020

\section{Palabras Clave}

Educacion juridica, pedagogía legal, didáctica legal, aprendizaje, conocimiento.

\section{Key Words}

Legal education, legal pedagogy, legal didactics, learning, knowledge

\section{DOI}

https://doi.org/10.35286/veritas. v21i1.257

\begin{abstract}
RESUMEN
Sin lugar a dudas en los últimos 75 años, desde la II guerra mundial, el mundo ha cambiado sobre manera, las comunicaciones, la ciencia, la medicina, la tecnología, la sociedad, la economía y por supuesto la educación no sería la excepción. En el campo del Derecho en el Perú también ha habido cambios, han aparecido nuevas instituciones jurídicas y nuevos hechos reales que cómo fuente del Derecho, nos hacen replantear la forma en que el Derecho se concibe y se debe enseñar en un mundo globalizado y tecnificado; por tanto, en el Perú, no se puede seguir enseñando de la misma manera, ni aprendiendo como se hacía hace más de 200 años desde el inicio de nuestra vida republicana y democrática en el Perú o en América Latina desde las facultades de Derecho. El Derecho se transforma y cambia, por ende debe cambiar la forma de enseñar y aprender.
\end{abstract}

\begin{abstract}
Sin lugar a dudas en los últimos 75 años, desde la II guerra mundial, el mundo ha cambiado sobre manera, las comunicaciones, la ciencia, la medicina, la tecnología, la sociedad, la economía y por supuesto la educación no sería la excepción. En el campo del Derecho en el Perú también ha habido cambios, han aparecido nuevas instituciones jurídicas y nuevos hechos reales que cómo fuente del Derecho, nos hacen replantear la forma en que el Derecho se concibe y se debe enseñar en un mundo globalizado y tecnificado; por tanto, en el Perú, no se puede seguir enseñando de la misma manera, ni aprendiendo como se hacía hace más de 200 años desde el inicio de nuestra vida republicana y democrática en el Perú o en América Latina desde las facultades de Derecho. El Derecho se transforma y cambia, por ende debe cambiar la forma de enseñar y aprender.
\end{abstract}

\section{INTRODUCCIÓN}

El presente articulo tiene como finalidad contribuir a una mejora en la formación de los docentes de Derecho en la ciudad de Arequipa, el Perú y de los futuros abogados, analizando de manera objetiva y dinámica, bajo una mirada crítica y propositiva el modelo educativo de enseñanza aprendizaje del Derecho actual. Para lo cual abordaremos la problemática metodológica, pedagógica y didáctica del Derecho en algunas facultades del Perú, teniendo en cuenta que esta es una investigacion parcial de posterior investigación mas profunda en el campo, como línea de investigación juridica en la cual los autores se encuentra involucrados, impartiendo clases en pre grado y post grado ademas de la capacitación y formación constante de docentes en Derecho en la que se han visto involucrados. Es un trabajo arduo y paciente, pues a lo largo de esta noble tarea, nos hemos podido dar cuenta que el perfil del docente abogado que enseña, desde hace varios años en las facultades de Derecho del país es dificilmente permeable a modificar su metodología de enseñanza

Correspondencia:

Neil Tejada-Pacheco

ntejada@ucsm.edu.pe aprendizaje clásica, mas por un tema de falta docilidad y humildad académica, tiempo y desconocimento. No asi las nuevas generaciones de abogados docentes, quienes siendo jovenes, inclusive nativos digitales o millenials, desean emprender el fascinante mundo de la docencia universitaria de cara al mundo globalizado y las nuevas TICs (Tecnologías de la información y la comunicación) que camina a pasos agigantados y de la cual el Derecho no puede permanecer indiferente. Es por ello que urge formar y acompañar desde la óptica de las nuevas corrientes pedagógicas y didácticas innovadoras el aprendizaje y la enseñanza de la ciencia jurídica basada en competencias y habilidades juridicas.

\section{METODOLOGÍA}

En el presente articulo base de investigacion juridica se aplico, desde la tradicion positivista (Durkheim.1938) y fenomenologica juridica, se aplico por ende una Metodologia Mixta: Teorica Practica: Cualitativa -empirica factica de observacion y analisis de contenido- (Taylor \& Bogdan, 1987) hermeneutico y de entrevista a docentes y alumnos de siete facultades de Derecho de Arequipa y una de Lima en Peru. Cuantitativa - inductiva fundamentado a partir de elementos exploratorios, descriptivos y explicativos (basados en informacion grafica, estadisticas, base de datos y bibliografia (Hernandez 1991. 2003). Como Metodo de 
Investigacion Juridica se aplicaron el Historico-CronologicoLogico, de Analisis-Sintesis e Inductivo-Deductivo.

\section{RESULTADOS}

Como muestra del presente articulo de investigacion se tomaron como referencia 8 facultades de Derecho de la ciudad de Arequipa Peru y 1 de Lima, arrojando los siguientes resultados:

- El 90\% de facultades de Derecho de la ciudad de Arequipa favorecen el uso de la clase magistral como metodologia o tecnica eficaz de enseñanza aprendizaje del Derecho. El $80 \%$ utiliza cañon proyector y presentaciones power point para trasmitir informacion en sus clases.

- Solo uno $30 \%$ recurre a otras metodologias de enseñanza aprendizaje como resolucion de problemas que considera casos de estudio. Solo un $20 \%$ utiliza las TICs como herramientas para promover el proceso de enseñanza aprendizaje en el alumno. En el $90 \%$ de los casos se recurre al uso de la pizarra acrilica para escribir o transcribir informacion complementaria al uso de Ppt.

- El 90\% aproximadamente utiliza el examen escrito como evaluacion final de conocimientos con carácter cognitivo y procedimental. Muy poco el saber actitudinal. Solo un $20 \%$ aproximadamente implementa formacion pedagogica y didactica especializada para los docentes de la carrera de Derecho.

- Suficientes datos muestran la necesidad de que la educación jurídica necesita realizar cambios en cómo se enseña y cómo aprende el alumnado. Es necesario mudarnos de un paradigma por objetivos a otro basado en un enfoque por competencias que enfatice, entre otros aspectos: a. El cambio en el ejercicio de roles de los y las docentes y del estudiantado. b. La capacitación del profesorado en temas de didáctica, pedagogía y evaluación de la enseñanza. c. La vinculación de la enseñanza con el contexto real. d. El uso de recursos y herramientas tecnológicas y de las redes. e. Una enseñanza más práctica, en la que medie la teoría, el uso de la tecnología de la información y el contexto. f. La preocupación real y profunda de las carreras de Derecho en desarrollar planes y programas con perfiles de ingreso y egreso congruentes con la realidad que se vive y con una dosis de visión.

- Ver Anexo 4

\section{DISCUSIÓN}

\section{Cap. I La enseñanza del Derecho en los siglos XIX y XX.}

El Perú se apresta a celebrar este 28 de julio del 2021, 200 años de vida republicana e independiente. Muchos cambios han sucedido desde que el General José de San Martin proclamo la independencia, la ilustración, la era industrial y la modernidad daban inicio a un continente americano que se independizaba de los reinos de España y Portugal asi como en Norteamerica de Inglaterra, con excepción de Canada.

Pasaron pues la 1era guerra mundial, la 2da., la guerra fria y el mundo se inicio en la era de la post modernidad con la caida del comunismo en la Union Sovietica, el Muro de Berlin y el gigante Chino y su cambio de modelo económico comunista estatal al de economía abierta donde ademas de los tigres asiaticos y la apertura de los mercados internacionales por medio de los TLCs de los cuales casi ningún país que se precie de ser parte de la globalización y el mercado, han logrado posicionarse como líderes no solo en lo económico sino en la innovación, el desarrollo tecnológico y de las telecomunicaciones.

La educación universitaria y las mismas universidades no se han quedado a la saga. Algo diferente ha ocurrido con la educación jurídica en America Latina heredera del sistema romano germánico, donde parece que no ha habido muchos cambios en el modelo de enseñanza aprendizaje y la currícula que no ha cambiado mucho desde hace dos siglos. Como parte de la post modernidad se han incorporado materias como los MARCs (Medios Alternativos de Resolucion de Conflictos), el Derecho Informático, el Derecho de la Propiedad Intelectual y Mercantil Internacional como lo mas novedoso.

En lo que se refiere al ámbito metodologico parece que en la enseñanza del Derecho no ha habido muchos cambios pues en muchas facultades de Derecho aún se sigue enseñando de la misma forma, por medio de la clase magistral que no es un método de enseñanza aprendizaje sino una tecnica pedagógica, se sigue aprendiendo de forma memoristica y se sigue evaluando de la misma manera por medio de un examen escrito. A lo sumo se han incorporado algunos elementos didácticos como el cañon multimedia, la pizarra de acrílico, los plumones en lugar de las tizas o gises, al acceso a internet para usar algunas veces videos de youtube o proyectar algunos materiales bibliográficos en el mejor de los casos.

Durante el siglo XIX la enseñanza del Derecho se baso en la clase magistral (donde el maestro era el principal actor del conocimiento por medio de su experiencia, conocimiento de la meteria y su locuacidad) y la casuística explicada (la idea de casuística se utiliza en el terreno de la ética aplicada para aludir a un análisis de distintos casos específicos que son esperables en un cierto contexto, en este caso el jurídico. De este modo la casuística se diferencia de aquellos razonamientos que se basan en reglas o principios. Dicho de otra manera, lo que hace la casuística es considerar casos particulares para la resolución de dilemas morales, encargándose de la aplicación de las reglas en circunstancias concretas. Los preceptos morales, por lo tanto, son aplicados a situaciones específicas provocadas por la acción del ser humano y no algo abstracto).

El modelo prusiano europeo, que luego de la caída de la monarquía y la aparición de la industria, propugnaba formar ciudadanos con formación militar e industrial, obedientes y trabajadores, pero que no se les permitiera pensar autónomamente sino obedecer, mucho menos innovar o desarrollar la creatividad, inclusive la conformación del salón de clases tenia connotaciones industriales y de orden militar, una butaca o pupitre detrás de otro, una gran pizarra al frente y al costado el escritorio del profesor con una silla (catedra) desde donde debía impartir su clase y claro con dos momentos de recreo previo al toque de un timbre que anunciaba dos momentos de descanso y uno de salida, esto en la escuela con sus diferencias y similitudes en la universidad del siglo XX y el siglo XXI donde el modelo de enseñanza aprendizaje se basa en competencias y el desarrollo de las TICs.

Así se formaron generaciones de generaciones de abogados que debían memorizar las leyes, códigos y si acaso la jurisprudencia, eso sí con mucha teoría y con poca práctica 
ya que en los últimos semestres debía corroborarse con la misma a nivel pre profesional en un estudio o despacho jurídico o en algún juzgado o fiscalía del Ministerio Publico para familiarizarse con la praxis litigiosa, que dicho sea de paso debía ser por antonomasia, el ejercicio profesional, por excelencia, de todo egresado de la facultad de Derecho.

Cap. II EI modelo de enseñanza aprendizaje del Derecho al momento en el Perú Siglo XX y XXI.

En la década de los 60s se produjo en América Latina un movimiento de reforma de la enseñanza del Derecho que duro aproximadamente hasta la decada de los 70 s que se llamo el período de reforma encabezado por la facultad de Derecho de la PUCP (Pontificia Universidad Católica del Perú y de la UNMSM (Universidad Nacional Mayor de San Marcos que por entonces ern las dos unicas facultades de Derecho mas relevantes en Lima, en Arequipa existian las facultades de Derecho de la UNSA (Universidad Nacional de San Agustin) y la de la UCSM (Universidad Católica de Santa Maria) privada (Zolezzi, 2017).

En la década de los 60s los estudios de Derecho duraban 5 años y todos los cursos eran obligatorios.

Por ejemplo el plan de estudios de la PUCP era el siguiente: (Ver Anexo 1)

Como se verá, los contenidos no son muy diferentes a los que se seguian enseñando y se siguen enseñando en muchas facultades de Derecho a las que hoy en dia el número de años de estudio se completa en 6 años mas uno de práctica pre profesional o el famoso SECIGRA (El Servicio Civil de Graduandos SECIGRA DERECHO es la práctica preprofesional que brinda al estudiante de las facultades y escuelas de Derecho del país, la oportunidad de conocer a través del ejercicio jurídico, el quehacer en la administración pública y de justicia. La referencia hacia el término servicio civil, hace hincapié en la naturaleza de servicio hacia los ciudadanos) (DL 26113).

Hoy por ejemplo la facultad de Derecho de la UCSM y de la UNSA 2018 en Arequipa maneja el siguiente plan de estudios o silabo: Ver el plan de estudios de la UNSA y UCSM: (Anexo 2 y 3 )

Como se podrá ver no se incluye ninguna materia en Metodología o Pedagogía y Didáctica del Derecho, como si lo incorpora la Facultad de Derecho de la Universidad de Chile4 esto es muy importante porque si bien es cierto que no todos los egresados de las facultades de Derecho se dedicaran a la docencia universitaria, con toda seguridad algunos si lo harán y de ellos dependerá la formación de los futuros abogados del País. Tampoco se incorporan en el silabo cursos en Inteligencia Emocional y Resiliencia Jurídica, Kinésica Jurídica (El comportamiento no verbal jurídico)5 https://analisisdeconducta.com/ https://behaviorandlaw. com/congreso2019/ fundamental para ejercer la abogacía conjuntamente con la argumentación jurídica (que curiosamente si se contempla) la cual solo se trata de manera general y no especifica cómo debería ser o por materias.
Cap. III Los nuevos modelos de enseñanza aprendizaje del Derecho basados en competencias y habilidades juridicas.

Al dia de hoy existen mas de 40 nuevas metodologias de enseñanza aprendizaje en el ámbito universitario o de educación superior de acuerdo a los reportes efectuados por el Observatorio de Innovación Educativa del Tecnológico de Monterrey5 (una de las mas prestigiosas universidades privadas de America Latina y a nivel global). Pero en el Perú, en la mayoría de las facultades de Derecho y Postgrado solo se utilizan casi siempre: La clase magistral, la casuística (no el método de casos) que a menudo se confunde con el aprendizaje basado en problemas, las exposisiones orales y grupales y como recursos técnicos didácticos las presentaciones power point, algunos audios y el cañon multimedia además de la pizarra de acrilico para algunas anotaciones. He notado que en algunas facultades de Derecho de Arequipa se utilizan algunos recursos procesales judiciales como: expedientes archivados o en curso, demandas civiles o denuncias penales, algunas sentencias e inclusive algunas jurisprudencias vinculadas al tema etc. No esta mal, pero no debemos olvidar que el ente jurisdiccional al admitir una demana, o una denuncia o dictar una sentencia o conformar un expediente no tiene fin pedagogico sino procesal, muy distinto del ambiente que se desarrolla en clase, por lo que si bien son recursos didacticos juridicos adecuados, es el profesor o docente quien debera "traducir" este material, para que pueda ser utilizado en clase con fin de enseñar y aprender pero en forma didactica y pedagogica. La enseñanza superior se basa hoy en el desarrollo de competencias (Saber, saber hacer y saber ser) a las que se incorporan las Tecnologías de la Información y la comunicación y la educación a distancia.

Mencionaremos algunas metodologias de enseñanza aprendizaje que deberian utilizarse y conocerse en el ambito de la enseñanza aprendizaje del Derecho que se emplean actualmente en muchas facultades de Derecho de universidades tanto de Norteamerica como Europa y que no se conocen bien (o se confunden) o no se utilizan en algunas facultades de Derecho peruanas y menos arequipeñas:

1. Aprendizaje Basado en el Metodo de casos. Estudio de casos reales metodologicamente escritos y expuestos en una sesion plenaria.

2. Aprendizaje Colaborativo. Diferente del llamado y muy usado "Trabajo grupal". Trabajo en equipo asignando a cada miembro un rol especifico a desarrollar dentro del grupo en forma metodica.

3. Aprendizaje Basado en la Investigacion Juridica. Aplicación de la investigacion factica de impacto no para obtener grado academico sino para desarrollar innovacion juridica.

4. Aprendizaje Basado en Diseño de Pensamiento. Llamado Design Thinking, siguiendo la dinamica del pensamiento humano para solucionar problemas diversos de la sociedad (en este caso juridicos). Con 5 pasos bien determinados.

5. Aprendizaje Basado en la Jurisprudencia. Desarrollo de habilidades de pensamiento critico basados en el estudio y la interpretacion de los principales fallos judiciales a nivel de ultima instancia.

6. Aprendizaje Basado en las TICs. Utilizacion de las tecnologias de la informacion aplicadas al ambito 
del Derecho. Hoy existe una importante influencia del llamado "Legal Tech" y la Inteligencia Artificial Juridica en el mundo globalizado.

7. Aprendizaje Invertido (Flipped Class). Modelo de clase invertida donde las "tareas" se desarrollan en clase y el estudio en casa.

8. Aprendizaje Social. Aprendizaje basado en la participacion del alumno en diversos compromisos de caracer social (aquí entra a tallar de manera excelente la labor de los estudios juridicos gratuitos de las facultades de Derecho y/o los laboratorios juridicos en diversas instituciones.

9. Aprendizaje Basado en Debates y Dilemas Eticos Juridicos. Desarrollo de debates en clase con temas relevantes de carácter juridico. Aquí los modelos de la ONU o la EOA sob un claro ejemplo. Los dilemas eticos juridicos que se abordan de manera similar al Metodo de Casos, son muy eficaces para formar la conciencia etica o deontologica del estudiante,

10. Aprendizaje Basado en el Pensamiento Critico. Desarrollo del pensamiento critico juridico analitico a partir de la realidad de acontecimientos o hechos de carácter juridico

11. Aprendizaje Basado en las Inteligencias Multiples. Basado en la propuesta de Howard Gardner en la que se sugiere que no todos aprendemos de la misma manera sino por medio de 8 diferentes tipos de inteligencia (o muchas mas) que nos permiten aprender de manera significativa y trascendente a partir del modo en que cada uno aprende.

12. Aprendizaje Basado en la Argumentacion Juridica. Desarrollo de la capacidad de comunicarse efectivamente y sustentar la postura juridica a partir de una adecuada formulacion de la teoria del caso. Considero que esta debe ser especializada y no generica como hasta el momento se viene impartiendo, ya que no es lo mismo argumentar penal que civilmente, administrativa o fiscalmente.

Cap. IV De un modelo de enseñanza basado en la clase magistral y la evaluacion cuantitativa a un modelo basado en la innovacion, la creatividad juridica y las competencias globales integrales.

Por varios cientos de años la clase magistral ha sido la herramienta por excelencia al momento de transmitir los conocimientos de la ciencia juridica. Y no es que este mal, pero teniendo en cuenta los ultimos avances en neuropedagogia, el estudio de los canales de aprendizaje, la taxonomia de Bloom.6, los niveles de atencion, la moderna pedagogia y la didactica entre otros, no se puede seguir privilegiando la clase magistral en la enseñanza del Derecho.

Hoy en dia la educacion superior, e inclusive la educacion a nivel secundaria privilegia el desarrollo dela innovacion y el emprendimiento, la creatividad que como decia Sir Ken Robinson en su famosa charla TED "Las escuelas matan la creatividad" 7 se requiere de un cambio de paradigma educativo que permita a los alumnos de pre grado e inclusive post grado desarrollar la innovacion por medio del ejercicio del pensamiento disrruptivo o divergente 8 juridico, puesto que los problemas sociales, institucionales juridicos reales deben ser abordados hoy en dia desde la solucion innovadora $\mathrm{y}$ creativa juridica.
Otro elemento importante que debe abordarse desde la globalizacion contemporanea es precisamente el papel del Derecho y del abogado en un mundo cada vez mas globalizado tanto en el mundo del comercio, los Derechos humanos, el medio ambiente y la proteccion de las comunidades postergadas inclusive el tema migratorio y sus vinculos con el Derecho Internacional Privado.

Cap. V De la propuesta de enseñanza aprendizaje del Derecho, la globalizacion y las competencias.

Definitivamente las facultades de Derecho de nuestro pais deben cambiar sus modelos educativos o estaran condenadas a seguir produciendo abogados a granel, abogados memoristas, amantes solo de la litis y no de las soluciones previas al problema legal o de la cultura de paz, la innovacion y la creatividad.

Hoy existe la tendencia en las diferentes universidades y en especial de las facultades de Derecho de no solo contratar a docentes abogados basados solo en su prestigio profesional, sus conocimientos en la materia o sus años de ejercicio profesional, sino que deban tener por lo menos una formacion pedagogica minima que les permita llegar a las aulas con herramientas competitivas basadas en la pedagogia $i$ la didactica. Es mas hoy en dia se sugiere que cada facultad o escuela profesional deba tener un asesor pedagogico especializado en la carrera ya sea ingenieria, arquitectura, medicina etc.

Pocas facultades de Derecho en el Perú buscan internacionalizar y certificar sus carreras ante organismos internacionales ad hoc con la finalidad de llevar a cabo intercambios academicos e incorporarse al ambito profesional desde una perspectiva global que les permita alcanzar dobles grados o titulaciones a nivel de pre grado o maestria, pero en el Peru esta forma aun es nueva y no dudo de que sera una heramienta muy eficaz para desarrollar competencias legales no solo locales, nacionales sino internacionales de los futuros abogados.

Según algunas investigaciones efectuadas por el autor establece que la mayoría de docentes abogados entraron a la docencia universitaria ya sea por invitación o concurso de meritos en los cuales no se requiere demuestren competencias y habilidades pedagogicas, pues estas se dan por sentado cuando se requiere "experiencia docente" en el curriculum vitae del postulante, cuando creo que deberia almenos exigirse de uno a dos años de formacion en pedagogia a los mismos y una vez dentro del claustro universitario continuar con su proceso de actualizacion y capacitacion continua a traves de programas de desarrollo de habilidades docentes que al efecto se implementen en las universidades que los contratan, ya sean estas publicas o privadas.

Cap. VI EI LegalTech y las TICs aplicadas al campo de las competencias y habilidades juridicas del siglo XXI.

Es innegable que la tecnologia tambien ha llevado a influir en el conocimiento y ejercicio del Derecho desde hace unos 15 años aproximadamente y con mucha fuerza. La Tecnologia legal a la que Podríamos definir la- o el- legaltech como un área del derecho nueva cuyo objeto es la mejora del derecho práctico en general a través de la implantación de tecnologías digitales. Proviene del término inglés "Legal Technology". En efecto, cuando hablamos de legaltech, hablamos de algo legal, algo jurídico, un área jurídica. Es 
importante aclarar este matiz, ya que podría entenderse, siguiendo la misma regla, que cuando hablamos de legaltech, hablamos de algo techie- tecnológico- y, por ende, se trataría de un área más de la ingeniería informática, por ejemplo. En este sentido, lo que hace que la legaltech sea un área del derecho y no de la tecnología es su contenido esencial o su objeto: la mejora del derecho.

Hoy la mayoría de sistemas procesales jurídicos en el mundo y más aún en América Latina, se vienen incorporando los expedientes digitales y los procesos electrónicos donde ya no es necesario que el abogado se desplace a la mesa de partes de la Corte o el juzgado para presentar un escrito o una demanda, sino que desde la comodidad de su estudio o despacho pueda presentar demandas, denuncias, escritos de toda índole, recibir notificaciones etc. $\mathrm{E}$ inclusive revisar el expediente desde su computadora, Tablet o Smartphone. Existen muchas aplicaciones para sistema Apple o Android que le permiten al abogado estar informado las 24 horas del día con el quehacer jurídico. Es mas hoy en día en algunos países como Canadá ya se cuenta con inteligencia artificial jurídica (abogados robot como el caso de "Ross" diseñado por la Universidad de Toronto. O el caso de China que utiliza IA (Inteligencia Artificial) para resolver algunos casos como el famoso robot Xiaofa en Beijing es uno de los pasos que las cortes en China están haciendo para crear "Cortes Inteligentes". Junto con incorporar robótica, los tribunales están usando intensivamente inteligencia artificial, big data y, recientemente, blockchain en los procedimientos judiciales. Todo esto nos hace pensar que en nuestras facultades de Derecho no se debe impedir el uso de las TICs por parte de los alumnos so pretexto de que los alumnos se distraen o no tienen la madurez necesaria para poder utilizarla, cuando en el futuro cercano esto ya es una realidad que debe ser abordada como una competencia fundamental en el proceso de enseñanza aprendizaje del Derecho.

\section{Apreciación final}

Lo que falta incorporar en la enseñanza aprendizaje del Derecho en las facultades de Derecho: Inteligencia emocional, Resiliencia y La Axiologia Juridica.

La calidad de la educación jurídica en Peru tiene una repercusión directa en la calidad de los servicios profesionales y la eficiencia del sistema jurídico peruano. De ahí, la importancia de que los y las docentes se preparen con estrategias didácticas, con propuestas de mejora en metodologías de enseñanza y de conocimientos del Derecho para formar a los y las estudiantes con las competencias específicas. La enseñanza en las carreras de Derecho debe considerar que las nuevas generaciones tienen características diferentes y construyen los conocimientos con la inclusión de la práctica en contextos reales. El desarrollo de las habilidades en el Sistema de Justicia se realiza conectando la teoría y la práctica, con el fin de hacer significativo el aprendizaje. Los modelos de enseñanza aprendizaje se manejan tradicionalmente y solo se han incorporado algunas herramientas teconologicas como el cañon multimedia, las ppt. Proyeccion de videos en clase. Planteamiento de problemas que llaman casos, sin un adecuado conocimiento del funcionnamiento metodologico o tecnico de enseñanza aprendizaje muchas veces confundiendo metodologias, tecnicas, instrumentos didacticos, Recientemente el uso de rubricas de evaluacion han sido incorporadas por exigencia de la SUNEDU para el proceso de acreditacion de las carreras entre otros requerimientos. Otro elemento a considerar es que si bien existen por parte de algunas facultades de Derecho interes por mejorar el proceso de enseñanza aprendizaje la forma de evaluar los aprendizajes sigue siendo la misma en su mayoria: por medio del un examen escrito que solo contempla saber cognitivo memoristico o en el mejor de los casos algo de saber procedimental pero casi nunca actitudinal. Finalmente y no menos importante dentro del proceso de enseñanza aprendizaje del Derecho en forma integral es la educación emocional, resiliente y la atención plena y consciente de la experiencia presente. Ocurre que en la universidad no se nos enseña como afrontar la frustración, los problemas de la vida personal y profesional, no se nos enseña a manejar las desgracias humanas como la enfermedad, la muerte o cosas menos graves como un despido, un fracaso sentimental o profesional. Rescato esto porque el abogado es una persona como cualquier otra, que sueña, rie, llora, se enoja, se frustra o deprime como cualquier otro profesional. La carga y presión laboral en el siglo XXI influye negativamente si no se sabe administrar. Nuestras sociedades occidentales y en especial las latinas no son tolerantes al error ni al fracaso. Existen numerosos estudios que contemplan la formación y el desarrollo profesional que incorpore la Inteligencia Emocional como una forma de dominio y control de nuestras emociones; de la resiliencia positiva, como una forma e manera la tragedia, la desgracia, la enfermedad etc.como parte de la vida humana; otro elemento importante sera el poder manejar y administrar el presente sacandole el maximo provecho al presente sin vivir del pasado como una carga que se arrastra y que condiciona el presente o vivir temeroso del futuro dejando de vivir el presente con toda su riqueza y vivencia personal.

Ser y no parecer!... No cabe duda que el ejercicio profesional del abogado, quizas mas que otras profesiones, esta sujeto a multiples situaciones que involucran, querramos o no, la salud emocional, psicologica y axiologica tan venida a menos con los ultimos escandalos vividos en diversas instituciones juiridicas de nuestro pais. El modelo educativo debe incorporar no solo desde la reflexión teórica deontologica juridica la formación en valores sino muy por el contrario el ejercicio y la reflexión práctica por medio de dilemas éticos jurídicos en todas las disciplinas, desde las eminentemente teoricas hasta las de la practica forense, pasando por la imagologia juridica (estudio y manejo de la imagen personal e institucional y la percepcion ciudadana de las mismas para incidir positivamente) y lo que hoy se ha denominado como "Behavior and law" o comportamiento no verbal juridico abordando temas como la corrupcion y el comportamiento etico juridico. Hoy mas que nunca la noble profesion del abogado se ha visto mas desacreditada que nunca encontrandose entre las profesionaes mas desprestigiadas de los ultimos tiempos no solo en el Perú sino en la mayoría de paises de América Latina, por lo que urge un nuevo paradigma no solo a nivel universitario sino familiar que es donde realmente se forma eticamente el ciudadano contrariamente como lo afirma el filosofo y pensador español Fernando Sabater que afirma que la educación debe formar tales.13 Solo asi lograremos preparar futuros abogados, jueces, fiscales, magistrados probos y competentes no solo en lo cognitivo sino en lo significativo del valor juridico que tanto necesita nuestra sociedad peruana. 


\section{REFERENCIAS BIBLIOGRÁFICAS}

1. Bigg, Jhon. (2006) Calidad del aprendizaje universitario. Segunda edicion Madrid. Narcea.

2. Boloña, C. (2000). Experiencias para una Economía al Servicio de la Gente. Escuela Nueva SA.

3. Castillo Freyre, Mario y Ricardo Vasquez Kunze (2005) La reforma de la enseñanza juridica en debate. Llima: Foro Academico.

4. Comision de Plan de Estudios de Derecho (2011) Propuestas de Plan de Estudios de Pregrado en Derecho PUCP. Lima. Pontificia Universidad Catolica del Peru.

5. Cuneo Macchiavello, Andres y Gonzlo Figueroa Yañez (1971) Las reformas operadas en la enseñanza del Derecho en Chile. Boletin del Instituto de Docencia e Investigacion Juridicas.

6. Decreto Ley $\mathrm{N}^{\circ} 26113$, Normas relativas al servicio civil de graduandos SECIGRA Derecho. Ley N ${ }^{\circ} 27687$, Ley que otorga carácter facultativo al SECIGRADERECHO y modifica artículos del Decreto Ley 26113. Reglamento de la Ley del Servicio Civil de Graduandos DECRETO SUPREMO No 009-2014-JUS.

7. Trazegnies, Fernando de, Jorge Avendaño V. y Lorenzo Zolezzi (1971) Nuestra reforma de la enseñanza del Derecho. Derecho PUCP 29. 132-149.
8. Turull Rubinat, Max. (2011). Experiencias de mejora e innovacion docente en el ambito del Derecho. Octaedro. ICE-UB España. Colección Educacion Universitaria.

9. Turull Rubinat, Max y Enoch Alberti Rovira (EDS). (2016) Octaedro. ICE.UB España. Colección Educacion Universitaria.

10. Villabella Armengol, Carlos Manuel. (2015) "Metodos de investigacion juridica. Algunas precisiones" Autor. Acerbo de la Biblioteca Juridica Virtual del Instituto de Investigaciones Juridicas de la UNAM Mexico.

11. Villapando, Jose Manuel. (2018). Enseñanza del Derecho y vocacion del abogado. Alegato y Defensa. Editorial Porrua Mexico. Escuela Libre de Derecho.

12. Weber, Max (1964) Economia y Sociedad. Mexico D.F Fondo de Cultura Economica.

13. Zolezzi Ibarcena, Lorenzo. "La enseñanza del Derecho" Colección lo escencial del Derecho 7 . Fondo Editorial de Derecho PUCP. 2017. Pag 13.

\section{ANEXOS}

\section{Anexo 1:}

\begin{tabular}{|c|c|c|c|c|}
\hline Primer año & Segundo Año & Tercer Año & Cuarto Año & Quinto Año \\
\hline Moral Cristiana & Sociologia Catolica. & $\begin{array}{c}\text { Derecho Canonico y Publico } \\
\text { Eclesiastico. }\end{array}$ & Deontologia Forense. & Filosofia del Derecho. \\
\hline $\begin{array}{c}\text { Introduccion a las ciencias } \\
\text { Juridicas }\end{array}$ & $\begin{array}{c}\text { Derecho Procesal Civil. Primer } \\
\text { curso. }\end{array}$ & $\begin{array}{l}\text { Derecho Procesal civil. } \\
\text { Segundo curso y practica. }\end{array}$ & $\begin{array}{l}\text { Derecho Procesal Civil. Tercer } \\
\text { curso y practica. }\end{array}$ & \\
\hline $\begin{array}{l}\text { Derecho Civil - Titulo } \\
\text { preliminar y personas }\end{array}$ & Derecho Civil. Derechos reales. & $\begin{array}{c}\text { Derecho Civil. Acto juridico y } \\
\text { obligaciones. }\end{array}$ & Derecho Civil. Contratos. & Derecho Civil. Sucesiones. \\
\hline $\begin{array}{c}\text { Derecho Penal - Primer curso. } \\
\text { Parte general. }\end{array}$ & $\begin{array}{l}\text { Derecho Penal: Segundo curso. } \\
\text { Parte especial y practica. }\end{array}$ & Derecho Procesal Penal. & Derecho Civil. Familia. & Practica procesal penal. \\
\hline Derecho Romano. Primer curso. & - Derecho Romano 2do curso & Derecho del Trabajo. & Practica de Derecho Registral. & Derecho Internacional Privado. \\
\hline \multirow[t]{6}{*}{ Economia Politica. } & Derecho Administrativo. & & Derecho Comercial. & Derecho Intenacional Publico. \\
\hline & $\begin{array}{c}\text { Derecho Constitucional general } \\
\text { del Peru }\end{array}$ & Historia del Derecho Peruano. & Derecho Tributario. & Derecho Maritimo. \\
\hline & Economia monetaria y bancaria & Medicina legal. & & Derecho Minero. \\
\hline & & & & Derecho Rural. \\
\hline & & & & Contabilidad Mercantil. \\
\hline & & & & Instrucción Pre Militar. \\
\hline
\end{tabular}

\section{Anexo 2:}

Plan de estudios de la carrera de Derecho de la UNSA Universidad Nacional de San Agustin Arequipa 2017 http://fde.unsa.edu.pe/plan-de-estudios/

\section{Anexo 3:}

Plan de estudios de la carrera de Derecho de la UCSM Universidad Catolica de Santa Maria 2016 h t t p s : // www.ucsm.edu.pe/derecho/plan-estudios-derecho/ 
VÉRITAS Vol. 21 N¹ (2020) 45-51

Anexo 4:

\begin{tabular}{|c|c|c|c|}
\hline $\begin{array}{l}\text { Universidad - Facultad de Derecho con } \\
\text { promedio de } 15 \text { a } 60 \text { alumnos por clase. }\end{array}$ & Metodologia de enseñanza y/o Tecnica. & $\begin{array}{l}\text { Aplican nuevos modelos de innovacion en la } \\
\text { enseñanza aprendizaje del Derecho. }\end{array}$ & $\begin{array}{l}\text { Porcentaje de enseñanza tradicional vs. } \\
\text { Modelos de innovacion docente enfocados a } \\
\text { competencias y habilidades juridicas. }\end{array}$ \\
\hline UNSA. Universidad Nacional de San Agustin & $\begin{array}{l}\text { Principalmente la clase magistral. } \\
\text { Estudio de casos (problemas). Ayudas } \\
\text { tecnologicas como cañon proyector. Power } \\
\text { Point. Lectura asignada a los alumnos. } \\
\text { Proyeccion de videos. } \\
\text { Exposicion por parte de los alumnos. } \\
\text { Trabajos grupales e individuales. } \\
\text { Examenes orales y escritos. }\end{array}$ & $\begin{array}{l}\text { Uso de plataformas digitales. Moodle. } \\
\text { Easyclass para subir informacion. }\end{array}$ & $90 \%-10 \%$ \\
\hline UCSM Universidad Catolica de Santa Maria. & $\begin{array}{l}\text { Principalmente la clase magistral. } \\
\text { Estudio de casos (problemas). Ayudas } \\
\text { tecnologicas como cañon proyector. Power } \\
\text { Point. Lectura asignada a los alumnos. } \\
\text { Proyeccion de videos. } \\
\text { Exposiciones grupales y personales. } \\
\text { Examenes orales y escritos. } \\
\text { Practicas pre profesionales. }\end{array}$ & $\begin{array}{l}\text { Uso de plataformas digitales. Para lista de } \\
\text { asistencia. }\end{array}$ & $80 \%-20 \%$ \\
\hline UCSP Universidad Catolica San Pablo. & $\begin{array}{l}\text { Principalmente la clase magistral. } \\
\text { Estudio de casos (problemas). Ayudas } \\
\text { tecnologicas como cañon proyector. Power } \\
\text { Point. Lectura asignada a los alumnos. } \\
\text { Proyeccion de videos. } \\
\text { Exposiciones grupales y personales. } \\
\text { Examenes orales y escritos. } \\
\text { Uso de TICs. } \\
\text { Practicas pre profesionales. }\end{array}$ & Uso de plataformas digitales. Moodle. & $70 \%-30 \%$ \\
\hline UTP. Universidad Tecnologica del Peru. & $\begin{array}{c}\text { Principalmente la clase magistral. } \\
\text { Estudio de casos (problemas). Ayudas } \\
\text { tecnologicas como cañon proyector. Power } \\
\text { Point. Lectura asignada a los alumnos. } \\
\text { Proyeccion de videos. } \\
\text { Exposiciones grupales y personales. } \\
\text { Examenes orales y escritos. } \\
\text { Uso de TICs. } \\
\text { Practicas pre profesionales. }\end{array}$ & $\begin{array}{l}\text { Uso de plataformas digitales. } \\
\text { Incorporacion de laboratorios juridicos. }\end{array}$ & $70 \%-30 \%$ \\
\hline UC. Universidad Continental. & $\begin{array}{c}\text { Principalmente la clase magistral. } \\
\text { Estudio de casos (problemas). Ayudas } \\
\text { tecnologicas como cañon proyector. Power } \\
\text { Point. Lectura asignada a los alumnos. } \\
\text { Proyeccion de videos. } \\
\text { Exposiciones grupales y personales. } \\
\text { Examenes orales y escritos. } \\
\text { Uso de TICs. }\end{array}$ & $\begin{array}{l}\text { Uso de plataformas digitales para tomar } \\
\text { asistencia. }\end{array}$ & $70 \%-30 \%$ \\
\hline UAP. Universidad Alas Peruanas. & $\begin{array}{l}\text { Principalmente la clase magistral. } \\
\text { Estudio de casos (problemas). Ayudas } \\
\text { tecnologicas como cañon proyector. Power } \\
\text { Point. Lectura asignada a los alumnos. } \\
\text { Exposiciones grupales y personales. } \\
\text { Examenes orales y escritos. }\end{array}$ & $\begin{array}{l}\text { Registro de asistencia manual en la mayoria } \\
\text { de los casos. }\end{array}$ & $80 \%-20 \%$ \\
\hline ULS. Universidad La Salle. & $\begin{array}{l}\text { Principalmente la clase magistral. } \\
\text { Estudio de casos (problemas). Ayudas } \\
\text { tecnologicas como cañon proyector. Power } \\
\text { Point. Lectura asignada a los alumnos. } \\
\text { Exposiciones grupales y personales. } \\
\text { Examenes orales y escritos. }\end{array}$ & $\begin{array}{l}\text { Uso de plataformas digitales para tomar } \\
\text { asistencia. }\end{array}$ & $70 \%-30 \%$ \\
\hline UNCV. Universidad Nestor Caceres Velasquez. & $\begin{array}{l}\text { Principalmente la clase magistral. } \\
\text { Estudio de casos (problemas). Ayudas } \\
\text { tecnologicas como cañon proyector. Power } \\
\text { Point. Lectura asignada a los alumnos. } \\
\text { Exposiciones grupales y personales. } \\
\text { Examenes orales y escritos. }\end{array}$ & $\begin{array}{l}\text { Registro de asistencia manual en la mayoria } \\
\text { de los casos. }\end{array}$ & $90 \%-10 \%$ \\
\hline $\begin{array}{l}\text { PUCP. Pontificia Universidad Catolica del } \\
\text { Peru. }\end{array}$ & $\begin{array}{c}\text { Principalmente la clase magistral. } \\
\text { Estudio de casos (problemas). } \\
\text { Ayudas tecnologicas como cañon proyector. } \\
\text { Power Point. Prezzi. Lectura asignada a los } \\
\text { alumnos. } \\
\text { Proyeccion de videos. Peliculas. } \\
\text { Aprendizaje Colaborativo. } \\
\text { Examenes orales y escritos. } \\
\text { Uso de TICs. } \\
\text { Uso del ingles tecnico legal. } \\
\text { Practicas pre profesionales. }\end{array}$ & $\begin{array}{l}\text { Registro de asistencia digital y uso de } \\
\text { plataformas digitales de aprendizaje. }\end{array}$ & $60 \%-40 \%$ \\
\hline
\end{tabular}

